\title{
Genetic Analysis of 11 Polymorphic Isozyme Loci in Chestnut Species and Characterization of Chestnut Cultivars by Multi-locus Allozyme Genotypes
}

\author{
Hongwen Huang, Fenny Dane, and Joseph D. Norton \\ Department of Horticulture, Auburn University, Auburn, AL 36849 \\ Additional index words. Castanea crenata, C. dentata, C. mollissima, C. seguinii, isoelectric focusing, single-tree progeny, \\ interspecific crosses

\begin{abstract}
Allozyme polymorphism in chestnut (Castanea) species was investigated using isoelectric focusing in thin-layer polyacrylamide slab gels. Genetic analysis of the progenies of intraspecific crosses and interspecific $\mathrm{F}_{2} \mathrm{~S}$ and backcrosses $\left(\mathrm{BC}_{1} \mathrm{~s}\right)$ allowed the verification of 11 polymorphic isozyme loci from 11 enzyme systems. The following loci were defined: Acp, Adh, Est-1, Est-2, Est-5, Me, Prx-1, Prx-2, Prx-3, Skd-3, and Skd-4. All polymorphic loci behaved as single-locus Mendelian genes. $S k d$ showed unique species specificity. $S k d-1$ and $S k d-2$ were unique to the American chestnut $(C$. dentata Borkh.) and the European chestnut ( $C$. sativa Mill.), whereas $S k d-3$ and $S k d-4$ were unique to the Chinese chestnut $(C$. mollissima Bl.) and the Japanese chestnut (C. crenata Sieb.). Linkage analysis revealed linkage for three pairs of loci: $S k d$ 3/Skd-4, Est-1/Est-2, and Est-5/Prx-1. The single-tree progeny method was used successfully for isozyme genetic analysis. Forty-seven chestnut cultivars in six chestnut species were characterized using 12 isozyme loci and can be unambiguously identified by 12 multi-locus genotypes. The interspecific and geographic relationships among species were also discussed.
\end{abstract}

The genus Castanea includes several commercially important chestnut species that are widely grown from the subtropic to temperate zones around the world. The current American (Am) chestnut $(C$. dentata) breeding program has focused on successive backcrosses of the Am $\times$ Chinese $(\mathrm{Ch})$ chestnut (C. mollissima) hybrids to the Am chestnuts for chestnut blight resistance (Burnham, 1988). This has resulted in increased public interest in chestnut breeding, collecting germplasm, and evaluating genetic variation between chestnut species (Cochran, 1990; Rutter et al., 1990). However, in contrast to other woody plant species, there have been few investigations into the genetics of these species, mainly because of their long juvenile stage. To date, only nine simply inherited morphological traits have been reported, and it has been difficult to use these as genetic markers in breeding programs (Hebard, 1992; Jaynes, 1975).

Allozymes have been used extensively in other fruit and nut species as genetic markers for identifying cultivars (Cerezo et al., 1989; Degani et al., 1990; Jarret and Litz, 1986; Weeden and Lamb, 1985), confirming hybridity (Anderson et al., 1991; Torres et al., 1982), measuring genetic diversity of plant populations (Hamrick, 1989), verifying taxonomic relationships (DeWald et al., 1992), constructing linkage maps (Bernatzky and Tanksley, 1986; Tanksley and Rick, 1980b), and performing many other aspects of plant breeding programs (Tanksley and Orton, 1983). However, no allozyme genetic studies have been reported in $\mathrm{Ch}$ and Am chestnuts.

The purpose of the present study was to 1) establish allozyme markers for $C$. mollissima and $C$. dentata by progeny tests of intraand interspecific crosses and 2) assign the allozyme multilocus genotypes to 47 cultivars and currently available germplasm selections to aid parental selections in chestnut breeding programs.

Received for publication 20 Sept. 1993. Accepted for publication 3 Feb. 1994. Journal no. 11-933604 of the Alabama Agricultural Experiment Station. The cost of publishing this paper was defrayed in part by the payment of page charges. Under postal regulations, this paper therefore must be hereby marked advertisement solely to indicate this fact.

\section{Materials and Methods}

Plant material. Families derived from intra- and interspecific crosses were used for genetic studies. At the intraspecific level, $\approx 200$ plants were initially surveyed for allozyme polymorphism using 20 enzyme systems. Twenty-five widely grown Ch chestnut cultivars and 30 seguin chestnut (C. seguinii Dode.) selections from the China chestnut germplasm plantations, 20 Am chestnut selections, 10 Japanese chestnut (C. crenata) cultivars from Japan and Korea, and a few European chestnut (C. sativa) seedlings were included in the survey. Progenies obtained from six controlled crosses of $\mathrm{Ch}$ chestnut cultivars were used for genetic analysis of allozymes. Additionally, the single-tree progeny method of Gillet (1991) was used to test allozyme inheritance because of the high degree of self-incompatibility of chestnut species and availability of large progeny numbers. Open-pollinated seeds were randomly harvested from a parental tree.

For interspecific studies, $\mathrm{F}_{2}$ and $\mathrm{BC}_{1}$ families of $\mathrm{Am}$ and $\mathrm{Ch}$ chestnuts from the research farm of the American Chestnut Foundation in Virginia were used. The $\mathrm{F}_{2}$ family was derived from an $\mathrm{Am} \times \mathrm{Ch} \mathrm{F}_{1}$ sib cross and the $\mathrm{BC}_{1}$ families were derived from $\mathrm{BCs}$ to Am (Hebard, 1992).

Electrophoresis and isozyme staining. Most isozyme assays of the intraspecific crosses were conducted on mature seed tissue, while dormant winter buds were used for $\mathrm{F}_{2}$ and $\mathrm{BC}_{1}$ families of interspecific crosses. Because of poor resolution in seed extracts, peroxidase assays were always conducted on dormant winter buds. Tissue specificity and subcellular location of all isozymes were determined using leaves of different developmental stages, catkins, roots, summer vegetative buds, and pollen. The best isozyme definition was obtained in seed tissue and winter dormant buds, and these tissues gave identical phenotypes. Seed tissue or buds ( $\approx 50 \mathrm{mg}$ ) were homogenized by hand in a prechilled mortar and pestle with cold extraction buffer [ $1 \mathrm{mg}$ tissue $/ 5$ to $10 \mu \mathrm{l}$ buffer \#3 (Wendel and Weeden, 1989) modified slightly by reducing bovine serum albumin to $0.025 \%(\mathrm{w} / \mathrm{v})]$. Crude extracts were centrifuged for 5 min at $5000 \times g$ in a centrifuge (5415C Eppendorf; Brinkmann Instruments, Westbury, N.Y.). A 1- $\mathrm{mm}^{2}$ Whatman no. 3 filter paper was soaked in the supernatant and used as sample for 
isoelectric focusing. The high phenolic content of the chestnut tissue made visualization of certain enzyme systems in some tissues impossible.

The isoelectric focusing polyacrylamide slab-gel procedure of Mulcahy et al. (1981) was used in this study because of its high resolution and high sample number $(\approx 100$ samples per run). Serva ampholytes with a pH of 4 to 9 were used. Gels were assayed for acid phosphatase (ACP; EC 31.3.2), alcohol dehydrogenase (ADH; EC 1.1.1.1), aminopeptidase (AMP; EC 3.4.11.1), aspartate aminotransferase (AAT; EC 2.6.1.1), catalase (CAT; EC 1.11.1.6),
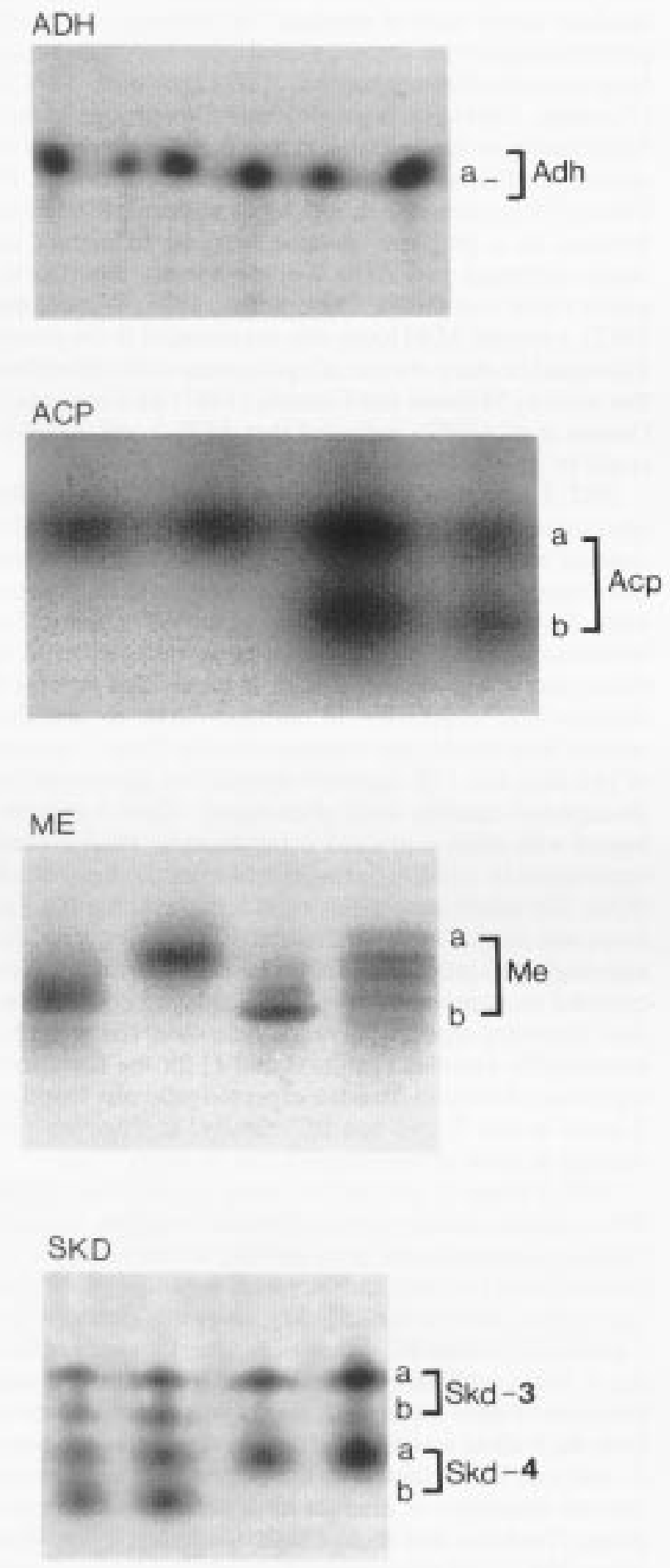

endopeptidase (ENP; EC 3.4.-.-), esterase (EST; EC 3.1.1), formate dehydrogenase (FDH; EC 1.2.1.2), $\beta$-galactosidase (GAL; EC 3.2.1.23), glucose-6-phosphate dehydrogenase (G6PDH; EC 1.1.1.49), glutamate dehydrogenase (GDH; EC 1.4.1.2), isocitrate dehydrogenase (IDH; EC 1.1.1.42), lactate dehydrogenase (LDH; EC 1.1.1.27), malate dehydrogenase (MDH; EC 1.1.1.37), malic enzyme (ME; EC 1.1.1.40), menadione reductase (MNR; EC 1.6.99.-), peroxidase (PRX; EC 1.11.1.7), 6-phosphogluconate dehydrogenase (PGD; EC 1.1.1.44), shikimate dehydrogenase (SKD; EC 1.1.1.25), and superoxide dismutase (SOD;EC 1.15.1.1).
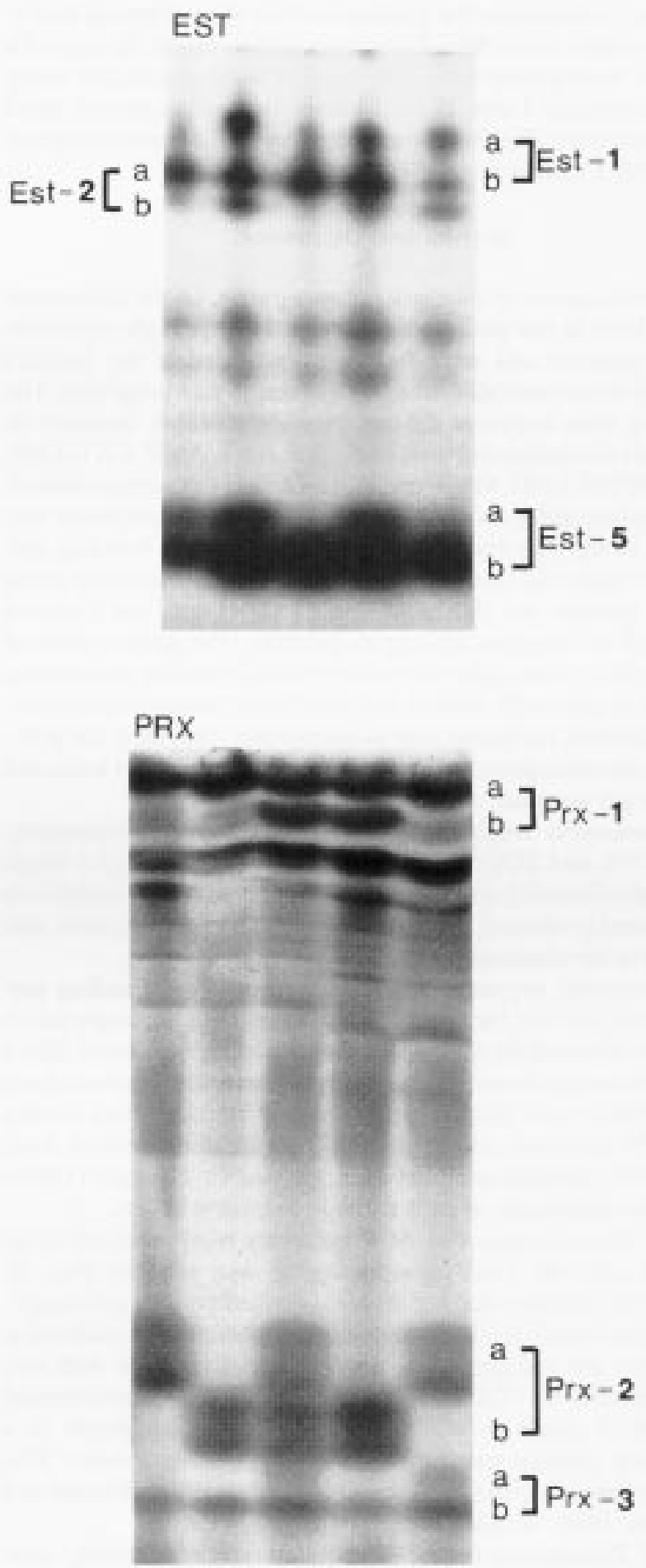

Fig. 1. Allele and locus designation and phenotypic segregation in six polymorphic enzymes in chestnut. Anode is toward the top. 
Staining recipes were those of Wendel and Weeden (1989) and Pasteur et al. (1988), with minor $\mathrm{pH}$ and ingredient concentration modifications.

Allozyme designation and data analysis. The genetic control of isozyme loci was postulated based on the phenotypic banding patterns and the segregation ratios of the controlled crosses. The loci for a given multiple-locus enzyme system were designated sequentially by number starting with 1 for the most anodal locus; at each locus, the alleles were designated sequentially by letter starting with $a$ for the most anodal allozyme. An allele lacking staining activity was designated with $n$ (null). Chi-square tests were used to determine the goodness-of-fit of segregating loci to expected single-locus Mendelian segregation ratios. In case of a two-class segregation ratio, chi-squares were calculated using Yates' correction (Yates, 1934). Linkage analyses were performed by the maximum likelihood method using the computer program LINKAGE-1 (Suiter et al., 1983).

\section{Results and Discussion}

General aspects of allozyme polymorphism. Of the 20 enzyme systems used in our preliminary survey, 11 gave high-resolution banding patterns and were further investigated in the families generated from controlled crosses or in single-tree progenies. The remaining nine enzymes did not provide scorable intensity or acceptable resolution and were excluded: AAT, AMP, CAT, ENP, GAL, G6PDH, LDH, MNR, and PGD. Of the 11 systems resolved, 7 were polymorphic. A considerable level of polymorphism was detected in the material examined. Representative banding patterns and allele and locus designation for all but one of the seven enzyme systems are shown in Fig. 1. MDH was not included because of its complex zymogram patterns. The genetic basis of this enzyme system could not be determined from the populations available in this study. Fifteen loci specifying twenty-eight alleles were identified, including four monomorphic enzymes. Six polymorphic enzymes provided an average of 2.18 alleles per locus and 1.83 loci per enzyme.

Monomorphic enzymes. Four enzymes were monomorphic. FDH, GDH, and SOD were invariant and each showed a single band in all chestnut populations. IDH displayed two intense bands with a weakly stained band occasionally, but no variation was detected in the chestnut populations.

Polymorphic enzymes. Variable, well-resolved banding patterns were observed for six enzyme systems (Fig. 1). Segregation data were obtained for eleven loci. Genetic interpretation of alleles for each locus was based on four types of populations derived from open-pollination of the single-tree progeny, the controlled crosses within $\mathrm{Ch}$ chestnut, and $\mathrm{F}_{2}$ and $\mathrm{BC}_{1}$ populations derived from interspecific hybridization between $\mathrm{Am}$ and $\mathrm{Ch}$ chestnut (Tables 1-4). The inheritance of each locus is discussed below.

$A C P$. Several regions of ACP isozymes were resolved in the gels, but only the most cathodal region was variable (Fig. 1). Segregating families derived from controlled crosses and singletree progenies demonstrated that the ACP phenotypes observed at this region are encoded by a single Mendelian locus with two alleles in chestnut (Tables 1-4). The observed ACP phenotypes consisted of single or double bands, a result that points to a monomeric enzyme structure for ACP in chestnut species. This was also found in other plant species (Kahler, 1983; Weeden and Robinson, 1986; Weeden et al., 1988).

$A D H$. Zymograms for ADH revealed only one activity zone (Fig. 1), designated as locus Adh. One intense band at pI $3.8(5 \mathrm{~mm}$ from origin) or the complete absence of this band at the same position (null allele) was observed. The data obtained from 10 families derived from intraspecific crosses and single-tree progenies and one $\mathrm{F}_{2}$ family from an interspecific cross were tested for their goodness of fit to the expected (presence-absence) segregation ratios. No significant deviations from the expected values were found (Tables 1-3). The segregation data only substantiate the presence of a putative null allele and do not indicate a quaternary enzyme structure. Therefore, two alleles are tentatively assigned at the $A d h$ locus: allele $A d h$ - $a$ coding for the presence of the band and a null allele $A d h-n$ coding for the absence of the band. The presence-absence mode was present in mature seeds and dormant winter buds of chestnut. The presence-absence banding patterns coded by an active and null allele have also been reported for peroxidase (Brewbaker et al., 1985; Rick et al., 1974), esterase (Tanksley, 1984), and $\beta$-galactosidase (Weeden and Marx, 1987). $\mathrm{ADH}$ has been demonstrated to be a dimeric enzyme in European chestnut and many other plants (Malvolti and Fineschi, 1987), but Torres (1974) reported an $A d h$ locus with an early (E) allele that behaved as a presence-absence isozyme in mature sunflower seeds. Although two ADH loci are usually reported in diploid plants (Arus and Orton, 1984; Suiter, 1988; Wendel and Parks, 1982), a second ADH locus was not revealed in the present study. This could be due to the use of a gel system with a $\mathrm{pH}$ different from that used by Malvolti and Fineschi (1987) for European chestnut. Gomes et al. (1982) indicated that ADH-2 and ADH-3 activity could be greatly inhibited at $\mathrm{pH} 6$.

EST. The best resolution and intensity of EST were obtained in seed tissue and dormant winter buds (Fig. 1). Four well-resolved zones of esterase activity were detected in seeds and dormant buds; three zones in young leaves, catkins, and bark; and two in summer buds. Mature leaves showed very little EST activity. Tissue and substrate specificity also have been demonstrated in apple (Manganaris and Alston, 1992). It seems that at least five loci regulate EST expression in chestnut. Both $\alpha$ - and $\beta$-naphthyl acetate were used in the staining solution. Zone 1 was comprised of two loci: Est-1 ( $\beta$-naphthyl-specific red phenotype) and Est-2 ( $\alpha$-naphthyl-specific black phenotype). Allele $b$ at locus 1 overlapped with allele $a$ at locus 2. Fortunately, the Est-1 red phenotypes could be reliably distinguished from the Est-2 black phenotypes. The inheritance of the $a$ and $b$ alleles at the Est- 1 and Est-2 locus was studied in 10 families derived from intraspecific crosses and single-tree progenies (Tables 1 and 2). The alleles at each locus encoded monomeric proteins and displayed codominant expression. Expected segregation ratios were observed in the $\mathrm{BC}_{1}$ and $\mathrm{F}_{2}$ interspecific families (Tables 3 and 4) for the Est-1 locus, but a significant deviation from the expected ratio was found at the Est2 locus in one $\mathrm{F}_{2}$ and one $\mathrm{BC}_{1}$ family. Segregation ratios were skewed in favor of the heterozygote genotype class.

EST-3 (zone 2) and EST-4 (zone 3) were also polymorphic. Two variants could be detected for each isozyme, but inconsistent banding expression and poor staining resolution made it difficult to score these two loci, and they were consequently excluded. The segregation analysis for EST-5 (zone 4) was similar to that of Est1 and Est-2 in three $\mathrm{BC}_{1}$ families and one $\mathrm{F}_{2}$ family in that a single Est-5 locus with two alleles ( $a$ and $b$ ) controlled monomeric enzymes (Tables 3 and 4). A third allele $c$ with a 2-mm mobility from the $b$ allele was detected only in single-tree progenies (Table 1) and can be attributed to the pollen source. Monomeric and dimeric structures of esterase have been reported in some other plants (Tanksley and Rick, 1980a; Tanksley, 1984; Weeden and Marx, 1987). Our data suggest that simple monogenic inheritance of these three esterases in chestnut species may be valuable in genetic studies and chestnut breeding programs. 
Table 1. Genetic analysis of 11 polymorphic isozyme loci using single-tree progeny in Castanea mollissima.

\begin{tabular}{|c|c|c|c|c|c|c|c|c|c|}
\hline \multirow[b]{2}{*}{ Locus } & \multicolumn{2}{|c|}{ Maternal tree $^{z}$} & \multicolumn{5}{|c|}{ Progeny genotype } & \multirow{2}{*}{$\begin{array}{c}\text { Expected progeny } \\
\text { relationship }\end{array}$} & \multirow[b]{2}{*}{$\chi^{2}$} \\
\hline & Name & Genotype & Total & aa & $\mathrm{ab}$ & $\mathrm{bb}$ & $\mathrm{bc}$ & & \\
\hline \multirow[t]{5}{*}{$\overline{A c p}$} & 1 & $\mathrm{bb}$ & 20 & 0 & 12 & 8 & & & \\
\hline & 2 & $a b$ & 17 & 3 & 10 & 4 & & $\mathrm{~N}_{\mathrm{ab}}=\mathrm{N}_{\mathrm{aa}}+\mathrm{N}_{\mathrm{bb}}$ & $0.24^{\mathrm{NS}}$ \\
\hline & 3 & $a b$ & 24 & 4 & 11 & 9 & & $\mathrm{~N}_{\mathrm{ab}}^{\mathrm{ab}}=\mathrm{N}_{\mathrm{aa}}^{\mathrm{aa}}+\mathrm{N}_{\mathrm{bb}}^{\mathrm{bb}}$ & $0.04^{\mathrm{NS}}$ \\
\hline & 4 & $\mathrm{bb}$ & 24 & 0 & 8 & 16 & & & \\
\hline & 5 & $a b$ & 24 & 5 & 9 & 10 & & $\mathrm{~N}_{\mathrm{ab}}=\mathrm{N}_{\mathrm{aa}}+\mathrm{N}_{\mathrm{bb}}$ & $1.04^{\mathrm{NS}}$ \\
\hline \multirow[t]{5}{*}{ Est-1 } & 1 & $\mathrm{ab}$ & 20 & 3 & 11 & 6 & & $\mathrm{~N}_{\mathrm{ab}}=\mathrm{N}_{\mathrm{aa}}+\mathrm{N}_{\mathrm{bb}}$ & $0.05^{\mathrm{NS}}$ \\
\hline & 2 & $\mathrm{bb}$ & 17 & 0 & 9 & 8 & & & \\
\hline & 3 & $a b$ & 24 & 4 & 11 & 9 & & $\mathrm{~N}_{\mathrm{ab}}=\mathrm{N}_{\mathrm{aa}}+\mathrm{N}_{\mathrm{bb}}$ & $0.04^{\mathrm{NS}}$ \\
\hline & 4 & $a b$ & 24 & 6 & 15 & 3 & & $\mathrm{~N}_{\mathrm{ab}}^{\mathrm{ab}}=\mathrm{N}_{\mathrm{aa}}^{\mathrm{aa}}+\mathrm{N}_{\mathrm{bb}}^{\mathrm{bb}}$ & $1.04^{\mathrm{NS}}$ \\
\hline & 5 & $a b$ & 24 & 4 & 13 & 7 & & $\mathrm{~N}_{\mathrm{ab}}^{\mathrm{ab}}=\mathrm{N}_{\mathrm{aa}}^{\mathrm{aa}}+\mathrm{N}_{\mathrm{bb}}^{\mathrm{bo}}$ & $0.04^{\mathrm{NS}}$ \\
\hline \multirow[t]{5}{*}{ Est-2 } & 1 & $\mathrm{ab}$ & 20 & 6 & 9 & 5 & & $\mathrm{~N}_{\mathrm{ab}}=\mathrm{N}_{\mathrm{aa}}+\mathrm{N}_{\mathrm{bb}}$ & $0.05^{\mathrm{NS}}$ \\
\hline & 2 & $\mathrm{bb}$ & 17 & 0 & 9 & 8 & & & \\
\hline & 3 & $a b$ & 24 & 6 & 14 & 4 & & $\mathrm{~N}_{\mathrm{ab}}=\mathrm{N}_{\mathrm{aa}}+\mathrm{N}_{\mathrm{bb}}$ & $0.38^{\mathrm{NS}}$ \\
\hline & 4 & $a b$ & 24 & 2 & 14 & 8 & & $\mathrm{~N}_{\mathrm{ab}}^{\mathrm{ab}}=\mathrm{N}_{\mathrm{aa}}^{\mathrm{a}}+\mathrm{N}_{\mathrm{bb}}$ & $0.38^{\mathrm{NS}}$ \\
\hline & 5 & $a b$ & 24 & 1 & 13 & 10 & & $\mathrm{~N}_{\mathrm{ab}}^{\mathrm{ab}}=\mathrm{N}_{\mathrm{aa}}^{\mathrm{aa}}+\mathrm{N}_{\mathrm{bb}}^{\mathrm{do}}$ & $0.04^{\mathrm{NS}}$ \\
\hline \multirow[t]{5}{*}{ Est-5 } & 1 & $\mathrm{bb}$ & 20 & 1 & 6 & 6 & 8 & & \\
\hline & 2 & $\mathrm{bb}$ & 17 & 1 & 7 & 6 & 4 & & \\
\hline & 3 & $\mathrm{bb}$ & 24 & 0 & 9 & 11 & 4 & & \\
\hline & 4 & $\mathrm{bb}$ & 24 & 0 & 2 & 13 & 9 & & \\
\hline & 5 & $\mathrm{bb}$ & 24 & 0 & 9 & 12 & 3 & & \\
\hline \multirow[t]{5}{*}{$M e$} & 1 & $\mathrm{bb}$ & 20 & 0 & 4 & 16 & & & \\
\hline & 2 & $\mathrm{bc}$ & 17 & 0 & 0 & 9 & 8 & $\mathrm{~N}_{\mathrm{bc}}=\mathrm{N}_{\mathrm{bb}}+\mathrm{N}_{\mathrm{cc}}$ & $0.00^{\mathrm{NS}}$ \\
\hline & 3 & $\mathrm{bb}$ & 22 & 0 & 6 & 16 & & & \\
\hline & 4 & $\mathrm{bb}$ & 22 & 0 & 0 & 22 & & & \\
\hline & 5 & $\mathrm{bb}$ & 24 & 0 & 0 & 24 & & & \\
\hline \multirow[t]{5}{*}{$\operatorname{Pr} x-1$} & 1 & $a b$ & 24 & 6 & 14 & 4 & & $\mathrm{~N}_{\mathrm{ab}}=\mathrm{N}_{\mathrm{aa}}+\mathrm{N}_{\mathrm{bb}}$ & $0.38^{\mathrm{NS}}$ \\
\hline & 2 & aa & 24 & 8 & 16 & 0 & & & \\
\hline & 3 & $a b$ & 24 & 8 & 15 & 1 & & $\mathrm{~N}_{\mathrm{ab}}=\mathrm{N}_{\mathrm{aa}}+\mathrm{N}_{\mathrm{bb}}$ & $1.04^{\mathrm{NS}}$ \\
\hline & 4 & aa & 24 & 10 & 14 & 0 & & & \\
\hline & 5 & aa & 24 & 7 & 17 & 0 & & & \\
\hline \multirow[t]{5}{*}{$\operatorname{Pr} x-2$} & 1 & $a b$ & 24 & 7 & 13 & 4 & & $\mathrm{~N}_{\mathrm{ab}}=\mathrm{N}_{\mathrm{aa}}+\mathrm{N}_{\mathrm{bb}}$ & $0.04^{\mathrm{NS}}$ \\
\hline & 2 & $a b$ & 24 & 8 & 10 & 6 & & $\mathrm{~N}_{\mathrm{ab}}^{\mathrm{ab}}=\mathrm{N}_{\mathrm{aa}}^{\mathrm{aa}}+\mathrm{N}_{\mathrm{bb}}^{\mathrm{bb}}$ & $0.38^{\mathrm{NS}}$ \\
\hline & 3 & $a b$ & 24 & 6 & 16 & 2 & & $\mathrm{~N}_{\mathrm{ab}}^{\mathrm{ab}}=\mathrm{N}_{\mathrm{aa}}^{\mathrm{ad}}+\mathrm{N}_{\mathrm{bb}}^{\mathrm{bo}}$ & $2.04^{\mathrm{NS}}$ \\
\hline & 4 & $a b$ & 24 & 8 & 12 & 4 & & $\mathrm{~N}_{\mathrm{ab}}^{\mathrm{ab}}=\mathrm{N}_{\mathrm{aa}}^{\mathrm{aa}}+\mathrm{N}_{\mathrm{bb}}^{\mathrm{do}}$ & $0.00^{\mathrm{NS}}$ \\
\hline & 5 & $a b$ & 24 & 14 & 7 & 3 & & $\mathrm{~N}_{\mathrm{ab}}^{\mathrm{ab}}=\mathrm{N}_{\mathrm{aa}}^{\mathrm{aa}}+\mathrm{N}_{\mathrm{bb}}^{\mathrm{bb}}$ & $3.38^{\mathrm{NS}}$ \\
\hline \multirow[t]{5}{*}{$\operatorname{Pr} x-3$} & 1 & $a b$ & 24 & 0 & 8 & 16 & & $\mathrm{~N}_{\mathrm{ab}}^{\mathrm{ab}}=\mathrm{N}_{\mathrm{aa}}^{\mathrm{ad}}+\mathrm{N}_{\mathrm{bb}}^{\mathrm{bo}}$ & $2.04^{\mathrm{NS}}$ \\
\hline & 2 & $a b$ & 24 & 0 & 15 & 9 & & $\mathrm{~N}_{\mathrm{ab}}=\mathrm{N}_{\mathrm{aa}}+\mathrm{N}_{\mathrm{bb}}$ & $1.04^{\mathrm{NS}}$ \\
\hline & 3 & $\mathrm{bb}$ & 24 & 0 & 4 & 20 & & & \\
\hline & 4 & $a b$ & 24 & 0 & 11 & 13 & & $\mathrm{~N}_{\mathrm{ab}}=\mathrm{N}_{\mathrm{aa}}+\mathrm{N}_{\mathrm{bb}}$ & $0.04^{\mathrm{NS}}$ \\
\hline & 5 & $\mathrm{bb}$ & 24 & 0 & 18 & 6 & & & \\
\hline \multirow[t]{5}{*}{$S k d-3$} & 1 & $\mathrm{bb}$ & 20 & 0 & 17 & 3 & & & \\
\hline & 2 & aa & 17 & 9 & 8 & 0 & & & \\
\hline & 3 & $a b$ & 24 & 1 & 16 & 7 & & $\mathrm{~N}_{\mathrm{ab}}=\mathrm{N}_{\mathrm{aa}}+\mathrm{N}_{\mathrm{bb}}$ & $2.04^{\mathrm{NS}}$ \\
\hline & 4 & $a b$ & 24 & 6 & 11 & 7 & & $\mathrm{~N}_{\mathrm{ab}}^{\mathrm{ab}}=\mathrm{N}_{\mathrm{aa}}^{\mathrm{aa}}+\mathrm{N}_{\mathrm{bb}}^{\mathrm{do}}$ & $0.04^{\mathrm{NS}}$ \\
\hline & 5 & $a b$ & 24 & 6 & 14 & 4 & & $\mathrm{~N}_{\mathrm{ab}}^{\mathrm{ab}}=\mathrm{N}_{\mathrm{aa}}^{\mathrm{aa}}+\mathrm{N}_{\mathrm{bb}}^{\mathrm{bb}}$ & $0.38^{\mathrm{NS}}$ \\
\hline \multirow[t]{6}{*}{$S k d-4$} & 1 & $\mathrm{bb}$ & 20 & 0 & 17 & 3 & & & \\
\hline & 2 & aa & 17 & 9 & 8 & 0 & & & \\
\hline & 3 & $\mathrm{ab}$ & 24 & 3 & 18 & 3 & & $\mathrm{~N}_{\mathrm{ab}}=\mathrm{N}_{\mathrm{aa}}+\mathrm{N}_{\mathrm{bb}}$ & $5.04^{*}$ \\
\hline & 4 & $a b$ & 24 & 8 & 8 & 8 & & $\mathrm{~N}_{\mathrm{ab}}^{\mathrm{ab}}=\mathrm{N}_{\mathrm{aa}}^{\mathrm{aa}}+\mathrm{N}_{\mathrm{bb}}^{\mathrm{do}}$ & $2.04^{\mathrm{NS}}$ \\
\hline & 5 & $a b$ & 24 & 8 & 11 & 5 & & $\mathrm{~N}_{\mathrm{ab}}=\mathrm{N}_{\mathrm{aa}}+\mathrm{N}_{\mathrm{bb}}$ & $0.04^{\mathrm{NS}}$ \\
\hline & & & & $a_{-}$ & oo & & & & \\
\hline$A d h$ & 1 & $a_{-}$ & 20 & $1 \overline{6}$ & 4 & & & $\mathrm{~N}_{\mathrm{a}} \geq \mathrm{N}_{\mathrm{oo}}$ & \\
\hline & 2 & $a_{-}$ & 17 & 11 & 6 & & & $\mathrm{~N}_{\mathrm{a}}^{\mathrm{a}} \geq \mathrm{N}_{\mathrm{oo}}$ & \\
\hline & 3 & $a_{-}$ & 24 & 16 & 8 & & & $\mathrm{~N}^{a-} \geq \mathrm{N}_{00}$ & \\
\hline & 4 & $a_{-}$ & 22 & 13 & 9 & & & $\mathrm{~N}_{\mathrm{a}}^{\mathrm{a}} \geq \mathrm{N}_{\mathrm{oo}}^{\mathrm{oo}}$ & \\
\hline & 5 & oo & 24 & 7 & 17 & & & & \\
\hline
\end{tabular}

${ }^{\mathrm{z}}$ Maternal tree: 1 = Cropper; 2 = Leader; 3 = Homestead; 4 = AU-60; 5 = AU-17.

${ }^{\mathrm{y}} \mathrm{N}=$ total number of progeny genotype.

Ns,*,*** Nonsignificant or significant at $P=0.05$ or 0.01 , respectively. 
Table 2. Single-locus segregation and chi-square test of 11 polymorphic loci in intraspecific progenies of Castanea mollissima.

\begin{tabular}{|c|c|c|c|c|c|c|c|c|c|}
\hline \multirow[b]{2}{*}{ Locus } & \multirow[b]{2}{*}{ Crosses $^{\mathrm{z}}$} & \multirow{2}{*}{$\begin{array}{c}\text { Parental } \\
\text { genotypes }\end{array}$} & \multicolumn{5}{|c|}{ Progeny } & \multirow{2}{*}{$\begin{array}{c}\text { Expected } \\
\text { ratio }\end{array}$} & \multirow[b]{2}{*}{$\chi^{2}$} \\
\hline & & & Total & aa & $a b$ & $\mathrm{bb}$ & $\overline{b c}$ & & \\
\hline \multirow[t]{5}{*}{$\overline{A c p}$} & 1 & $a b \times b b$ & 20 & --- & 10 & 10 & & $1: 1$ & $0.000^{\mathrm{NS}}$ \\
\hline & 2 & $\mathrm{bb} \times \mathrm{bb}$ & 19 & --- & 19 & --- & & & \\
\hline & 3 & $a b \times b b$ & 24 & --- & 8 & 16 & & $1: 1$ & $2.042^{\mathrm{NS}}$ \\
\hline & 4 & $\mathrm{bb} \times \mathrm{ab}$ & 19 & --- & 7 & 12 & & $1: 1$ & $0.842^{\mathrm{NS}}$ \\
\hline & 5 & $a b \times b b$ & 36 & --- & 22 & 14 & & $1: 1$ & $1.361^{\mathrm{NS}}$ \\
\hline \multirow[t]{5}{*}{ Est-1 } & 1 & $a b \times a b$ & 20 & 3 & 10 & 7 & & $1: 2: 1$ & $1.600^{\mathrm{NS}}$ \\
\hline & 2 & $\mathrm{bb} \times \mathrm{ab}$ & 19 & 5 & 9 & 5 & & $1: 2: 1$ & $0.053^{\mathrm{NS}}$ \\
\hline & 3 & $\mathrm{bb} \times \mathrm{ab}$ & 24 & --- & 12 & 12 & & $1: 1$ & $0.000^{\mathrm{NS}}$ \\
\hline & 4 & $a b \times b b$ & 19 & --- & 9 & 10 & & $1: 1$ & $0.000^{\mathrm{NS}}$ \\
\hline & 5 & $a b \times a b$ & 36 & 6 & 20 & 10 & & $1: 2: 1$ & $1.333^{\mathrm{NS}}$ \\
\hline \multirow[t]{5}{*}{ Est-2 } & 1 & $a b \times a b$ & 20 & 7 & 11 & 2 & & $1: 2: 1$ & $2.700^{\mathrm{NS}}$ \\
\hline & 2 & $a b \times a b$ & 19 & 4 & 10 & 5 & & $1: 2: 1$ & $0.157^{\mathrm{NS}}$ \\
\hline & 3 & $\mathrm{bb} \times \mathrm{ab}$ & 24 & --- & 10 & 14 & & $1: 1$ & $0.375^{\mathrm{NS}}$ \\
\hline & 4 & $a b \times b b$ & 19 & --- & 9 & 10 & & $1: 1$ & $0.000^{\mathrm{NS}}$ \\
\hline & 5 & $a b \times a b$ & 36 & 9 & 19 & 8 & & $1: 2: 1$ & $0.167^{\mathrm{NS}}$ \\
\hline \multirow[t]{5}{*}{ Est-5 } & 1 & $b b \times b b$ & 20 & --- & --- & 20 & & & \\
\hline & 2 & $\mathrm{bb} \times \mathrm{bb}$ & 19 & --- & --- & 19 & & & \\
\hline & 3 & $\mathrm{bb} \times \mathrm{bb}$ & 24 & --- & --- & 24 & & & \\
\hline & 4 & $\mathrm{bb} \times \mathrm{bb}$ & 19 & --- & --- & 19 & & & \\
\hline & 5 & $\mathrm{bb} \times \mathrm{bb}$ & 36 & --- & --- & 36 & & & \\
\hline \multirow[t]{5}{*}{$M e$} & 1 & $b b \times b b$ & 20 & --- & --- & 20 & & & \\
\hline & 2 & $\mathrm{bb} \times \mathrm{bb}$ & 19 & --- & --- & 19 & & & \\
\hline & 3 & $\mathrm{bc} \times \mathrm{bb}$ & 24 & --- & --- & 15 & 9 & $1: 1$ & $1.042^{\mathrm{NS}}$ \\
\hline & 4 & $b b \times b c$ & 19 & --- & --- & 13 & 6 & $1: 1$ & $1.895^{\mathrm{NS}}$ \\
\hline & 5 & $\mathrm{bb} \times \mathrm{bb}$ & 36 & --- & --- & 36 & & & \\
\hline \multirow[t]{5}{*}{$\operatorname{Pr} x-1$} & 1 & $a b \times a b$ & 24 & 8 & 14 & 2 & & $1: 2: 1$ & $3.667^{\mathrm{NS}}$ \\
\hline & 2 & $\mathrm{ab} \times \mathrm{aa}$ & 24 & 16 & 8 & --- & & $1: 1$ & $2.042^{\mathrm{NS}}$ \\
\hline & 3 & aa $x$ aa & 24 & 24 & --- & --- & & & \\
\hline & 4 & $\mathrm{ab} \times \mathrm{aa}$ & 24 & 14 & 10 & --- & & $1: 1$ & $0.375^{\mathrm{NS}}$ \\
\hline & 5 & $\mathrm{ab} \times \mathrm{aa}$ & 24 & 13 & 11 & --- & & $1: 1$ & $0.042^{\mathrm{NS}}$ \\
\hline \multirow[t]{5}{*}{$\operatorname{Pr} x-2$} & 1 & $a b \times a b$ & 24 & 5 & 15 & 4 & & $1: 2: 1$ & $1.583^{\mathrm{NS}}$ \\
\hline & 2 & $a b \times a b$ & 24 & 8 & 13 & 3 & & $1: 2: 1$ & $2.249^{\mathrm{NS}}$ \\
\hline & 3 & $a b \times a b$ & 24 & 4 & 16 & 4 & & $1: 2: 1$ & $2.667^{\mathrm{NS}}$ \\
\hline & 4 & $a b \times a b$ & 24 & 6 & 15 & 3 & & $1: 2: 1$ & $2.250^{\mathrm{NS}}$ \\
\hline & 5 & $a b \times a b$ & 24 & 5 & 12 & 7 & & $1: 2: 1$ & $0.333^{\mathrm{NS}}$ \\
\hline \multirow[t]{5}{*}{$\operatorname{Pr} x-3$} & 1 & $\mathrm{bb} \times \mathrm{ab}$ & 24 & --- & 8 & 16 & & $1: 1$ & $2.042^{\mathrm{NS}}$ \\
\hline & 2 & $a b \times a b$ & 24 & 10 & 10 & 4 & & $1: 2: 1$ & $3.667^{\mathrm{NS}}$ \\
\hline & 3 & $a b \times a b$ & 24 & 5 & 12 & 7 & & $1: 2: 1$ & $0.333^{\mathrm{NS}}$ \\
\hline & 4 & $a b \times a b$ & 24 & 4 & 10 & 10 & & $1: 2: 1$ & $3.667^{\mathrm{NS}}$ \\
\hline & 5 & $\mathrm{bb} \times \mathrm{ab}$ & 24 & --- & 9 & 15 & & $1: 1$ & $1.042^{\mathrm{NS}}$ \\
\hline \multirow[t]{5}{*}{$S k d-3$} & 1 & $a b \times b b$ & 20 & --- & 17 & 3 & & $1: 1$ & $8.450^{* * *}$ \\
\hline & 2 & $\mathrm{bb} \times \mathrm{ab}$ & 19 & --- & 9 & 10 & & $1: 1$ & $0.000^{\mathrm{NS}}$ \\
\hline & 3 & $\mathrm{aa} \times \mathrm{ab}$ & 24 & 15 & 9 & --- & & $1: 1$ & $1.042^{\mathrm{NS}}$ \\
\hline & 4 & $\mathrm{bb} \times \mathrm{aa}$ & 19 & --- & 9 & --- & & & \\
\hline & 5 & $a b \times a b$ & 36 & 3 & 18 & 15 & & $1: 2: 1$ & $8.000^{*}$ \\
\hline$S k d-4$ & 1 & $a b \times b b$ & 20 & --- & 9 & 11 & & $1: 1$ & $0.050^{\mathrm{NS}}$ \\
\hline & 2 & $\mathrm{bb} \times \mathrm{ab}$ & 19 & --- & 8 & 11 & & $1: 1$ & $0.211^{\mathrm{NS}}$ \\
\hline & 3 & $\mathrm{aa} \times \mathrm{ab}$ & 24 & 11 & 13 & --- & & $1: 1$ & $0.042^{\mathrm{NS}}$ \\
\hline & 4 & $\mathrm{bb} \times \mathrm{aa}$ & 19 & --- & 19 & --- & & & \\
\hline & 5 & $a b \times a b$ & 36 & 12 & 6 & 18 & & $1: 2: 1$ & $18.000^{* * *}$ \\
\hline & & & & $a_{-}$ & oo & & & & \\
\hline$A d h$ & 1 & ao $\mathrm{x}$ ao & 20 & $1 \overline{6}$ & 4 & & & $3: 1$ & $0.067^{\mathrm{NS}}$ \\
\hline & 2 & ao $\mathrm{x}$ ao & 19 & 15 & 4 & & & $3: 1$ & $0.018^{\mathrm{NS}}$ \\
\hline & 3 & ao $\mathrm{x}$ ao & 24 & 20 & 4 & & & $3: 1$ & $0.500^{\mathrm{NS}}$ \\
\hline & 4 & ao $\times$ ao & 19 & 15 & 4 & & & $3: 1$ & $0.018^{\mathrm{NS}}$ \\
\hline & 5 & ao $\mathrm{x}$ ao & 36 & 25 & 11 & & & $3: 1$ & $0.333^{\mathrm{NS}}$ \\
\hline
\end{tabular}

${ }^{\mathrm{z}}$ Cross 1 = Homestead $\times$ Cropper; 2 = Cropper $\times$ AU-60; 3 = Leader $\times$ AU-60; 4 = Cropper $\times$ Leader; 5 = Homestead $\times$ AU-60.

Ns,*,** Nonsignificant or significant at $P=0.05$ or 0.01 respectively. 
Table 3. Single-locus segregation and chi-square test of nine polymorphic loci in the $\mathrm{F}_{2}$ family from the interspecific cross Castanea mollissima $\mathrm{x} C$. dentata, R4T52 x R4T31.

\begin{tabular}{|c|c|c|c|c|c|c|c|}
\hline \multirow[b]{2}{*}{ Locus } & \multirow{2}{*}{$\begin{array}{l}\text { Parental } \\
\text { genotype }\end{array}$} & \multicolumn{4}{|c|}{ Progeny genotypes } & \multirow{2}{*}{$\begin{array}{c}\text { Expected } \\
\text { ratio }\end{array}$} & \multirow[b]{2}{*}{$\chi^{2}$} \\
\hline & & Total & aa & $a b$ & $\overline{\mathrm{bb}}$ & & \\
\hline$\overline{A c p}$ & $a b \times a b$ & 63 & 10 & 32 & 21 & $1: 2: 1$ & $3.857^{\mathrm{NS}}$ \\
\hline Est-1 & $\mathrm{bb} \times \mathrm{ab}$ & 63 & --- & 38 & 25 & $1: 1$ & $2.286^{\mathrm{NS}}$ \\
\hline Est-2 & $\mathrm{aa} \times \mathrm{ab}$ & 60 & 17 & 43 & --- & $1: 1$ & $10.417^{* *}$ \\
\hline Est-5 & $a b \times a b$ & 63 & 13 & 34 & 16 & $1: 2: 1$ & $0.683^{\mathrm{NS}}$ \\
\hline$M e$ & $a b \times a b$ & 63 & 13 & 20 & 30 & $1: 2: 1$ & $17.571^{\text {** }}$ \\
\hline $\operatorname{Pr} x-1$ & $a b \times a b$ & 63 & 19 & 33 & 11 & $1: 2: 1$ & $2.175^{\mathrm{Ns}}$ \\
\hline $\operatorname{Pr} x-2$ & $a b \times a b$ & 63 & 18 & 30 & 15 & $1: 2: 1$ & $0.428^{\mathrm{NS}}$ \\
\hline \multirow[t]{2}{*}{$\operatorname{Pr} x-3$} & $\mathrm{bb} \times \mathrm{ab}$ & 63 & --- & 22 & 41 & $1: 1$ & $5.143^{*}$ \\
\hline & & & $a_{-}$ & oo & & & \\
\hline$A d h$ & $a_{-} \times$oo & 48 & 25 & 23 & & $1: 1$ & $0.021^{\mathrm{NS}}$ \\
\hline
\end{tabular}

Table 4. Single-locus segregation and chi-square test of eight polymorphic loci in $\mathrm{BC}_{1}$ families from the interspecific cross of Castanea mollissima x C. dentata.

\begin{tabular}{|c|c|c|c|c|c|c|c|c|}
\hline \multirow[b]{2}{*}{ Locus } & \multirow[b]{2}{*}{ Crosses $^{\mathrm{z}}$} & \multirow{2}{*}{$\begin{array}{c}\text { Parental } \\
\text { Genotype }\end{array}$} & \multicolumn{4}{|c|}{ Progeny genotypes } & \multirow{2}{*}{$\begin{array}{c}\text { Expected } \\
\text { ratio }\end{array}$} & \multirow[b]{2}{*}{$\chi^{2}$} \\
\hline & & & Total & aa & $\mathrm{ab}$ & $\overline{\mathrm{bb}}$ & & \\
\hline \multirow[t]{3}{*}{$\overline{A c p}$} & 2 & $\mathrm{bb} \times \mathrm{ab}$ & 27 & --- & 9 & 18 & $1: 1$ & $2.370^{\mathrm{NS}}$ \\
\hline & 3 & $\mathrm{bb} \times \mathrm{ab}$ & 25 & --- & 16 & 9 & $1: 1$ & $1.440^{\mathrm{NS}}$ \\
\hline & 4 & $\mathrm{bb} \times \mathrm{ab}$ & 50 & --- & 24 & 26 & $1: 1$ & $0.020^{\mathrm{NS}}$ \\
\hline \multirow[t]{3}{*}{ Est-1 } & 2 & $\mathrm{bb} \times \mathrm{ab}$ & 27 & --- & 15 & 12 & $1: 1$ & $0.148^{\mathrm{NS}}$ \\
\hline & 3 & $\mathrm{bb} \times \mathrm{ab}$ & 25 & --- & 12 & 13 & $1: 1$ & $0.000^{\mathrm{NS}}$ \\
\hline & 4 & $\mathrm{bb} \times \mathrm{ab}$ & 50 & --- & 22 & 28 & $1: 1$ & $0.500^{\mathrm{NS}}$ \\
\hline \multirow[t]{3}{*}{ Est-2 } & 2 & $\mathrm{aa} \times \mathrm{ab}$ & 27 & 7 & 20 & --- & $1: 1$ & $5.294^{*}$ \\
\hline & 3 & $\mathrm{bb} \times \mathrm{aa}$ & 25 & --- & 25 & --- & & \\
\hline & 4 & $\mathrm{aa} \times \mathrm{ab}$ & 50 & 20 & 30 & --- & $1: 1$ & $1.620^{\mathrm{NS}}$ \\
\hline \multirow[t]{3}{*}{ Est-5 } & 2 & $\mathrm{bb} \times \mathrm{ab}$ & 27 & --- & 10 & 17 & $1: 1$ & $1.333^{\mathrm{NS}}$ \\
\hline & 3 & $a b \times a b$ & 25 & 5 & 14 & 6 & $1: 2: 1$ & $0.267^{\mathrm{NS}}$ \\
\hline & 4 & $a a \times a b$ & 50 & 23 & 27 & --- & $1: 1$ & $0.180^{\mathrm{NS}}$ \\
\hline \multirow[t]{3}{*}{$M e$} & 2 & $\mathrm{bb} \times \mathrm{ab}$ & 27 & --- & 11 & 16 & $1: 1$ & $0.593^{\mathrm{NS}}$ \\
\hline & 3 & $\mathrm{bb} \times \mathrm{ab}$ & 25 & --- & 10 & 15 & $1: 1$ & $0.640^{\mathrm{NS}}$ \\
\hline & 4 & $\mathrm{bb} \times \mathrm{ab}$ & 50 & --- & 30 & 20 & $1: 1$ & $1.620^{\mathrm{NS}}$ \\
\hline \multirow[t]{3}{*}{$\operatorname{Pr} x-1$} & 2 & $\mathrm{aa} \times \mathrm{ab}$ & 27 & 14 & 13 & --- & $1: 1$ & $3.278^{\mathrm{NS}}$ \\
\hline & 3 & $\mathrm{aa} \times \mathrm{ab}$ & 25 & 24 & 1 & --- & $1: 1$ & $19.360^{* *}$ \\
\hline & 4 & $\mathrm{aa} \times \mathrm{ab}$ & 50 & 22 & 28 & --- & $1: 1$ & $0.500^{\mathrm{NS}}$ \\
\hline \multirow[t]{3}{*}{$\operatorname{Pr} x-2$} & 2 & $\mathrm{aa} \times \mathrm{ab}$ & 27 & 13 & 14 & --- & $1: 1$ & $0.000^{\mathrm{NS}}$ \\
\hline & 3 & $a b \times a b$ & 25 & 15 & 5 & 5 & $1: 2: 1$ & $22.500^{* * *}$ \\
\hline & 4 & $\mathrm{aa} \times \mathrm{ab}$ & 50 & 21 & 29 & --- & $1: 1$ & $0.980^{\mathrm{NS}}$ \\
\hline \multirow[t]{3}{*}{$\operatorname{Pr} x-3$} & 2 & $\mathrm{bb} \times \mathrm{ab}$ & 27 & --- & 14 & 13 & $1: 1$ & $0.000^{\mathrm{NS}}$ \\
\hline & 3 & $a b \times b b$ & 25 & --- & 18 & 7 & $1: 1$ & $4.000^{*}$ \\
\hline & 4 & $\mathrm{bb} \times \mathrm{ab}$ & 50 & --- & 27 & 23 & $1: 1$ & $0.180^{\mathrm{NS}}$ \\
\hline
\end{tabular}

Crosses: 2 = American $\times$ R4T31; 3 = QA1 x R4T52; 4 = American $\times$ Nanking.

Ns, ${ }^{*}, * * N o n s i g n i f i c a n t$ or significant at $P=0.05$ or 0.01 respectively.

$M E$. ME exhibited only one activity zone. Its one- vs. fivebanded phenotype can be interpreted as the expression of a tetrameric enzyme. The genetic control was best described by the action of a single Mendelian locus $(\mathrm{Me})$ consisting of three codominant alleles $a, b$, and $c$, because $a$ and $b$ alleles segregated normally in the $\mathrm{BC}_{1}$ families (Table 4) and the $b$ and $c$ alleles segregated normally in three intraspecifically derived families (Tables 1 and 2). Although a significant deviation was observed in the $\mathrm{F}_{2}$ family (Table 3 ), this could be considered as sampling error or preferential segregation in the $\mathrm{F}_{2}$ family of an interspecific cross. $\mathrm{ME}$ behaves electrophoretically as a tetrameric protein with genotype $M e-a a$ and $M e-b b$ for the homozygote and $M e-a b$ for the heterozygote (Fig. 1). ME has been reported to be tetrameric in apple (Weeden and Lamb, 1987) but monomeric in citrus (Torres et al., 1985).
$P R X$. Three main polymorphic activity zones were observed for PRX (Fig. 1). Single- and double-banded phenotypes were observed at zone- 1 and zone- 3 and suggest the monomeric and codominant nature of two functional loci: Prx-1 and Prx-3. Intraspecific and interspecific crosses between one- and twobanded parents yielded offspring with two phenotypic classes (similar to the parental classes) in a 1:1 ratio. A normal 1:2:1 segregation ratio was found when crosses were made between twobanded parents (Tables 1-4). No significant deviation from the expected segregation ratio was found in 10 intraspecific crosses and single-tree progenies and most interspecifically derived $\mathrm{BC}_{1}$ or $\mathrm{F}_{2}$ families, except one $\mathrm{F}_{2}$ and one $\mathrm{BC}_{1}$ family with marginal significance at the $5 \%$ level at the Prx-3 locus and one $\mathrm{BC}_{1}$ family with $1 \%$ significance at the Prx-1 locus. This could be considered 
due to chance alone because there was no consistent trend observed among other families. It can be concluded that Prx-1 and Prx-3 act as a single locus in Mendelian fashion with two active $a$ and $b$ alleles at each locus (Fig. 1). Prx-2 exhibited one- or two-doublet phenotypic patterns. Segregation analysis for Prx-2 (Tables 1-4), based on monogenic inheritance patterns, supports the hypothesis that a single locus codes for this monomeric doublet isozyme. Two alleles were detected at Prx-2, with allele $a$ coding for an anodal doublet and allele $b$ coding for a cathodal doublet. The heterozygote genotype Prx-2-ab expressed both doublets. PRX is one of most extensively investigated enzyme systems. In maize, as many as 13 peroxidases have been specified (Brewbaker et al., 1985). PRX has been reported to be monomeric in most plants (Garcia et al., 1982; Quiros and McHale, 1985; Quiros and Morgan, 1981; Rick et al., 1974; Torres, 1980; Weeden and Marx, 1987; Weeden and Robinson, 1986; Weeden et al., 1988) and frequently to be expressed as presence-absence phenotype (Brewbaker et al., 1985), doublet (Berg and Wijsman, 1982), or triplet (Hirano and Nagunuma, 1979). However, PRX has not been investigated extensively in chestnut species. Sawano et al. (1984) concluded, based on their study of three chestnut species, that there was no special differentiation of peroxidase isozymes among Castanea spp. Anagnostakis (1991) reported that an unspecified PRX anodal locus, which behaved as a monomeric isozyme, could be equivalent to the Prx-1 in this study. It seemed, based on our germplasm survey, that $a$ and $b$ alleles at $\operatorname{Pr} x-1$ have almost equal gene frequencies in $C$. mollissima, but that the $a$ allele is predominant in $C$. dentata and the $b$ allele is predominant in $C$. seguinii. Intraspecific and interspecific hybridization could generate sufficient polymorphism at this locus for it to be used as a genetic marker.

$S K D$. This enzyme exhibited a unique species-specific zymogram for $C$. mollissima and $C$. dentata. Two relatively anodal SKD activity zones were observed and designated as SKD-1 and SKD2 for $C$. dentata. Either single- or double-banded patterns were detected at both zones. The two bands at the SKD- 1 have $\mathrm{pI}$ values of 4.9 and 5.1 (10 and $11 \mathrm{~mm}$ from origin), respectively, whereas the two bands at SKD-2 have pI values of 5.4 and 5.6 (13 and 14 $\mathrm{mm}$ from origin). SKD-1 and SKD-2 seemed to be regulated by two separate single loci, tentatively designated as $S k d-1$ and $S k d$ 2 , with alleles $a$ and $b$ at each locus governing monomeric enzymes. Because $C$. dentata is almost extinct, sufficient progenies from controlled crosses within pure $C$. dentata can hardly be obtained and allelic segregation at the $S k d-1$ and $S k d-2$ locus cannot be analyzed in this species.

In C. mollissima, two different zones cathodal to SKD-2 were observed. One zone, designated as SKD-3, showed two different bands of pI $5.6(14 \mathrm{~mm})$ and $5.8(15 \mathrm{~mm})$. The other zone, SKD-
4, showed two bands of pI 6.0 (16 mm) and 6.2 (17 mm) (Fig. 1). The most-anodal band at SKD-3 overlapped with the most cathodal band of SKD-2 at pI 5.6 (14 mm from origin). Genetic analysis was carried out in families of controlled crosses and single-tree progeny (Tables 1 and 2). Segregation data supported the monogenic inheritance of monomeric enzymes SKD-3 and SKD-4 in $C$. mollissima. SKD-3 and SKD-4 are governed by two loci, $S k d-3$ and $S k d-4$. SKD has been known to be a regular monomeric enzyme, and one or two loci are usually reported in diploid plant species (Navot and Zamir, 1986; Quiros and McHale, 1985; Weeden and Gottlieb, 1980; Weeden and Robinson, 1986). Although monomeric doublet phenotypes have been found in pepper (Tanksley, 1984), celery (Arus and Orton, 1984), and maize (Wendel et al., 1988), the clear banding phenotypes resolved by the high-resolution isoelectric focusing system used in this study suggested that SKD was expressed as an one- or two-banded monomeric enzyme in chestnut species, even if the bands are closely spaced $(\approx 1 \mathrm{~mm}$ apart). The observed data also suggest a linkage between $S k d-3$ and $S k d-4$, because high frequencies of the same genotypes have occurred at both loci and significant deviations from the expected ratios were found in the controlled crosses and single-tree progenies (Tables 1 and 2).

In $C$. mollissima $\times C$. dentata hybrids, the $\mathrm{F}_{1}$ possessed the isozyme banding pattern of both parent species and displayed hybrid phenotypic patterns with four zones. The $\mathrm{F}_{2}$ population segregated into three isozyme phenotype pattern classes only: $C$. dentata with SKD-1, -2; hybrid with SKD-1,-2,-3,-4, and $C$. mollissima with SKD-3, -4 . This phenotypic segregation in the $\mathrm{F}_{2}$, based on a 1:2:1 Mendelian segregation ratio was significantly skewed in favor of $C$. mollissima phenotypes. However, three $\mathrm{BC}_{1}$ families segregated normally into two classes to fit the expected 1:1 ratio. Chi-square tests do not detect heterogeneity or significant deviations from the totals of all families. The overall data suggest that the SKD phenotypic patterns are inherited in a Mendelian fashion.

The eleven isozyme loci described above are inherited as single Mendelian genes in chestnut species. As neither Am nor Ch chestnuts have been subjected to a systematic investigation of allozyme variation, the results from this study are quite encouraging and can be used to evaluate the overall genetic diversity of the two species for germplasm collection and conservation. It may lead to a saturated gene linkage map and tagging genes of economically important traits (Bernatzky and Tanksley, 1986; Tanksley and Rick, 1980b). More allozyme polymorphism will be discovered by expanding additional enzyme systems and using morediverse germplasm. The presence of species-predominant alleles, such as Prx-1-aa in C. dentata and Prx-1-bb in C. seguinii, and

Table 5. Number of families analyzed by chi-square contingency tests for independent assortment of jointly segregating isozyme loci (above the diagonal) and number of families deviating from independent assortment (below the diagonal) in Castanea spp.

\begin{tabular}{|c|c|c|c|c|c|c|c|c|c|c|c|}
\hline Locus & $A c p$ & $A d h$ & Estl & Est2 & Est5 & $M e$ & $\operatorname{Prx} 1$ & $\operatorname{Prx} 2$ & prx3 & $S k d 3$ & Skd4 \\
\hline$\overline{A c p}$ & & 6 & 7 & 5 & 3 & 4 & 6 & 7 & 6 & 4 & 4 \\
\hline$A d h$ & 0 & & 5 & 5 & 1 & 3 & 5 & 6 & 5 & 4 & 4 \\
\hline Est 1 & 0 & 1 & & 5 & 3 & 5 & 6 & 7 & 8 & 4 & 4 \\
\hline Est2 & 0 & 1 & 3 & & --- & 2 & 4 & 5 & 5 & 4 & 4 \\
\hline Est 5 & 0 & 0 & 0 & --- & & 4 & 3 & 3 & 3 & --- & --- \\
\hline$M e$ & 0 & 0 & 0 & 0 & 0 & & 3 & 3 & 3 & 1 & 1 \\
\hline $\operatorname{Prxl}$ & 0 & 0 & 0 & 0 & 2 & 0 & & 4 & 4 & 3 & 3 \\
\hline $\operatorname{Pr} x 2$ & 0 & 0 & 0 & 0 & 0 & 0 & 0 & & 7 & 4 & 4 \\
\hline Prx3 & 0 & 0 & 0 & 0 & 1 & 0 & 0 & 0 & & 4 & 4 \\
\hline$S k d 3$ & 0 & 0 & 0 & 0 & --- & 0 & 0 & 0 & 0 & & 4 \\
\hline$S k d 4$ & 0 & 0 & 0 & 0 & --- & 0 & 0 & 0 & 0 & 4 & \\
\hline
\end{tabular}


Table 6. Assignment of multi-locus genotypes for chestnut (Castanea) cultivars.

\begin{tabular}{|c|c|c|c|c|c|c|c|c|c|c|c|c|}
\hline \multirow[b]{2}{*}{ Cultivar } & \multicolumn{12}{|c|}{ Genotype $^{z}$} \\
\hline & 1 & 2 & 3 & 4 & 5 & 6 & 7 & 8 & 9 & 10 & 11 & 12 \\
\hline \multicolumn{13}{|l|}{ C. mollissima } \\
\hline Jiazha & $a b$ & $a_{-}$ & $\mathrm{ab}$ & $a b$ & $a b$ & $\mathrm{bb}$ & aa & $\mathrm{bc}$ & oo & oo & $a b$ & aa \\
\hline Yangmaoli & $\mathrm{bc}$ & oo & $a b$ & $a b$ & $\mathrm{ab}$ & $\mathrm{ab}$ & aa & $a b$ & oo & oo & $\mathrm{ab}$ & aa \\
\hline Zaozhuang & $a b$ & $a_{-}$ & $\mathrm{bb}$ & $a b$ & $\mathrm{ab}$ & $\mathrm{ab}$ & aa & $\mathrm{bc}$ & oo & oo & aa & aa \\
\hline Liuyuebao & $\mathrm{bc}$ & $a_{-}$ & $a b$ & $a b$ & aa & $\mathrm{bb}$ & aa & $\mathrm{bb}$ & oo & oo & aa & aa \\
\hline Jianding & $\mathrm{bc}$ & oo & $\mathrm{ab}$ & $a b$ & aa & aa & aa & $\mathrm{bc}$ & oo & oo & $a b$ & aa \\
\hline Jiujiazhong & $\mathrm{bc}$ & oo & $\mathrm{ab}$ & aa & aa & aa & aa & $\mathrm{bb}$ & oo & oo & aa & aa \\
\hline Youli & $\mathrm{bb}$ & oo & $a b$ & aa & aa & $\mathrm{bb}$ & $\mathrm{ab}$ & $\mathrm{bb}$ & oo & oo & $a b$ & $a b$ \\
\hline Zhongcili & $\mathrm{bc}$ & oo & $a b$ & $\mathrm{aa}$ & $a b$ & $\mathrm{ab}$ & $\mathrm{aa}$ & $a b$ & oo & oo & $a b$ & aa \\
\hline Jinfeng & $a b$ & $a_{-}$ & $a b$ & $\mathrm{bb}$ & $\mathrm{ac}$ & $a b$ & $a b$ & $\mathrm{bb}$ & oo & oo & $\mathrm{bb}$ & aa \\
\hline Yanhong & $a b$ & oo & $\mathrm{ab}$ & $\mathrm{bb}$ & aa & $a b$ & aa & $\mathrm{bb}$ & oo & oo & aa & aa \\
\hline Hongguang & $\mathrm{bc}$ & oo & $\mathrm{ab}$ & aa & aa & $\mathrm{bb}$ & aa & $\mathrm{bc}$ & oo & oo & aa & aa \\
\hline Duancibanhong & $\mathrm{bc}$ & $a_{-}$ & $\mathrm{bb}$ & $\mathrm{ab}$ & aa & aa & aa & $\mathrm{ab}$ & oo & oo & $a b$ & aa \\
\hline Changcibanhong & $\mathrm{bb}$ & oo & $\mathrm{ab}$ & $\mathrm{bb}$ & aa & $a b$ & aa & $\mathrm{bb}$ & oo & oo & aa & aa \\
\hline Zhongguohongpi & $\mathrm{bb}$ & $a_{-}$ & $a b$ & $\mathrm{bb}$ & aa & $a b$ & $\mathrm{aa}$ & $\mathrm{bb}$ & oo & oo & aa & aa \\
\hline Homestead & $a b$ & $a_{-}$ & $\mathrm{ab}$ & $\mathrm{bb}$ & $\mathrm{bb}$ & $a b$ & $a b$ & $\mathrm{bb}$ & oo & oo & $a b$ & $a b$ \\
\hline Leader & $a b$ & $a_{-}$ & $\mathrm{bb}$ & $\mathrm{bb}$ & $\mathrm{bc}$ & aa & $\mathrm{ab}$ & $a b$ & oo & oo & aa & aa \\
\hline Cropper & $\mathrm{bb}$ & $a_{-}$ & $\mathrm{ab}$ & $\mathrm{bb}$ & $\mathrm{bb}$ & $a b$ & $\mathrm{ab}$ & $a b$ & oo & oo & $\mathrm{bb}$ & $\mathrm{bb}$ \\
\hline AU-17 & $a b$ & oo & $\mathrm{ab}$ & $\mathrm{bb}$ & $\mathrm{bb}$ & aa & $\mathrm{ab}$ & $\mathrm{bb}$ & oo & oo & $a b$ & $a b$ \\
\hline AU-60 & $\mathrm{bb}$ & $a_{-}$ & $a b$ & $\mathrm{bb}$ & $\mathrm{bb}$ & aa & $a b$ & $a b$ & oo & oo & $a b$ & $a b$ \\
\hline \multicolumn{13}{|l|}{ C. dentata } \\
\hline WS male sterile & oo & oo & $\mathrm{bb}$ & aa & oo & aa & aa & $\mathrm{bb}$ & $\mathrm{bb}$ & aa & oo & oo \\
\hline WS E1 & $a b$ & oo & $\mathrm{ab}$ & $\mathrm{ab}$ & $\mathrm{bb}$ & aa & aa & $\mathrm{bb}$ & aa & aa & oo & oo \\
\hline Hesper & aa & oo & $a b$ & aa & $\mathrm{bc}$ & aa & aa & $\mathrm{bb}$ & $a b$ & aa & oo & oo \\
\hline Shinebone & $\mathrm{bb}$ & oo & $\mathrm{ab}$ & $\mathrm{ab}$ & $\mathrm{bb}$ & $\mathrm{bb}$ & aa & $\mathrm{bb}$ & aa & aa & oo & oo \\
\hline \multicolumn{13}{|l|}{ C. crenata } \\
\hline Tanawa & oo & $a_{-}$ & $\mathrm{bc}$ & aa & aa & $\mathrm{bb}$ & aa & $\mathrm{bc}$ & oo & oo & $a b$ & aa \\
\hline Ibuki & $\mathrm{bb}$ & $a_{-}$ & $\mathrm{bc}$ & aa & aa & $\mathrm{bb}$ & aa & $\mathrm{ab}$ & oo & oo & aa & aa \\
\hline Toyotawa-wase & $\mathrm{bb}$ & $a_{-}$ & $\mathrm{ab}$ & $\mathrm{ab}$ & aa & $\mathrm{bb}$ & aa & $\mathrm{bb}$ & oo & oo & aa & aa \\
\hline Ginyose & oo & $a_{-}$ & $\mathrm{cc}$ & $\mathrm{bb}$ & aa & $\mathrm{bb}$ & aa & $\mathrm{bb}$ & oo & oo & $a b$ & aa \\
\hline Yinhong & $\mathrm{bb}$ & $a_{-}$ & $\mathrm{cc}$ & aa & aa & $a b$ & aa & $\mathrm{bb}$ & oo & oo & $a b$ & $a b$ \\
\hline Toshan 60 & $\mathrm{bb}$ & $a_{-}$ & $\mathrm{bc}$ & $\mathrm{bb}$ & $a b$ & $\mathrm{bb}$ & aa & $\mathrm{bb}$ & oo & oo & $a b$ & aa \\
\hline Toshan 9 & $a b$ & oo & $\mathrm{bc}$ & $\mathrm{bb}$ & $a b$ & $a b$ & aa & $a b$ & oo & oo & aa & aa \\
\hline \multicolumn{13}{|l|}{ C. sativa } \\
\hline Weich & $\mathrm{bc}$ & $a_{-}$ & $\mathrm{bc}$ & $a b$ & $a b$ & aa & aa & $a b$ & aa & aa & oo & oo \\
\hline Klepper & $\mathrm{cc}$ & oo & $\mathrm{bc}$ & aa & $\mathrm{ab}$ & oo & $\mathrm{aa}$ & $a b$ & aa & aa & oo & oo \\
\hline \multicolumn{13}{|l|}{ C. seguinii } \\
\hline H91-13 & $a b$ & oo & $\mathrm{bb}$ & $\mathrm{bb}$ & $a b$ & $a b$ & $a b$ & $a b$ & oo & oo & $a b$ & aa \\
\hline H91-6 & $a b$ & oo & $\mathrm{bb}$ & $a b$ & $a b$ & $\mathrm{bb}$ & aa & $\mathrm{bb}$ & oo & oo & $a b$ & aa \\
\hline H91-9 & $\mathrm{bb}$ & $a_{-}$ & $a b$ & $a b$ & $a b$ & $\mathrm{bb}$ & $a b$ & $a b$ & oo & oo & $a b$ & aa \\
\hline H91-3 & aa & $a_{-}$ & $\mathrm{ab}$ & $\mathrm{bb}$ & aa & $\mathrm{bb}$ & $\mathrm{bb}$ & $a b$ & oo & oo & $a b$ & aa \\
\hline H91-8 & aa & $a_{-}$ & $a b$ & $\mathrm{bb}$ & $a b$ & $\mathrm{bb}$ & $\mathrm{bb}$ & $a b$ & oo & oo & $a b$ & $a b$ \\
\hline H91-4 & $\mathrm{bb}$ & oo & $\mathrm{bb}$ & $a b$ & aа & $\mathrm{bb}$ & $\mathrm{bb}$ & $\mathrm{bb}$ & oo & oo & aa & aa \\
\hline H91-2 & $a b$ & $a_{-}$ & $\mathrm{ab}$ & $a b$ & aa & $\mathrm{bb}$ & $\mathrm{bb}$ & $\mathrm{bb}$ & oo & oo & aa & aa \\
\hline H91-5 & $\mathrm{bc}$ & $a_{-}$ & $a b$ & $a b$ & aa & $\mathrm{bb}$ & $a b$ & $a b$ & oo & oo & $a b$ & aa \\
\hline H91-7 & $\mathrm{ac}$ & $a_{-}$ & $a b$ & $\mathrm{bb}$ & aa & $\mathrm{bb}$ & $a b$ & $a b$ & oo & oo & $a b$ & $a b$ \\
\hline H91-10 & aa & $a_{-}$ & $a b$ & $\mathrm{aa}$ & aa & $\mathrm{bb}$ & $\mathrm{aa}$ & $\mathrm{bb}$ & oo & oo & aa & aa \\
\hline H91-79 & $\mathrm{bc}$ & oo & $a b$ & aa & aa & $\mathrm{bb}$ & $\mathrm{bb}$ & $\mathrm{bb}$ & oo & oo & aa & aa \\
\hline \multicolumn{13}{|l|}{ C. pumila } \\
\hline Am4065 & $\mathrm{bb}$ & oo & $\mathrm{ab}$ & $a b$ & $a b$ & aa & aa & $\mathrm{bb}$ & $a b$ & aa & oo & oo \\
\hline Am2348 & aa & oo & $\mathrm{ab}$ & $a b$ & aa & aa & aa & $a b$ & aa & aa & oo & oo \\
\hline Am2342 & aa & oo & oo & oo & aa & aa & $\mathrm{aa}$ & $a b$ & $a b$ & aa & oo & oo \\
\hline Am3532 & $\mathrm{ac}$ & oo & $a b$ & $a b$ & aa & aa & aa & $\mathrm{bb}$ & aа & $a b$ & oo & oo \\
\hline
\end{tabular}

${ }^{\mathrm{z}} 1=A c p ; 2=A d h ; 3,4=$ Est $-2,5 ; 5=$ Me $; 6,7,8=\operatorname{Prx}-1,2,3 ; 9,10,11,12=$ Skd-1, 2, 3, 4.

species-specific allozymes $(S k d)$ in different chestnut species can help generate sufficient polymorphism and segregation markers when interspecific hybridizations are made. SKD isozymes are particularly interesting in chestnut species. SKD-1 and SKD-2 are restricted to Am and European species, while SKD-3 and SKD-4 are restricted to $\mathrm{Ch}$ and Japanese species. The existence of this species-specific marker provides a unique tool for verifying interspecific hybrids and detecting species introgression. 
The single-tree progeny method (Gillet, 1991) was effective for the genetic analysis of chestnut isozymes. Disturbed segregation ratios $(P<0.05)$ were observed in $5.8 \%$ of the controlled crosses and $5.4 \%$ of the single-tree progeny. Such a frequency of segregation distortion can be attributed in both cases to chance alone. The single-tree progeny method is a convenient and effective alternative for isozyme genetic analysis in self-incompatible species, particularly in the preliminary stage, when large amounts of material are needed for detecting isozyme polymorphism.

Linkage analysis. Forty-two pairs of loci were tested for independent assortment (Table 5). Three pairs of isozyme loci with significant deviations from random assortment were identified: Skd-3 with $S k d-4$; Prx-1 with Est-5, and Est-1 with Est-2. A highly significant $(P<0.01)$ linkage between $S k d-3$ and $S k d-4$ was consistently observed in all four segregating families. A significant linkage was also observed in three of five families between Est -1 and Est-2 and in two of three families between Est-5 and Prx$1(P<0.05)$. In addition, significant departure from independent assortment $(P<0.05)$ occurred between the following pairs of loci: Est-1 and Adh, Est-2 and Adh, and Prx-3 and Est-5, each in one segregating family.

Characterization of chestnut cultivars by multi-locus genotypes. Theoretically, there are $\mathrm{n}^{\mathrm{m}}$ multi-locus genotype combinations available for cultivar discrimination, if we refer to $\mathrm{m}$ as the number of polymorphic loci and $\mathrm{n}$ as the average number of genotypes at each locus. However, the practical number of combinations is less than $\mathrm{n}^{\mathrm{m}}$ due to the presence of predominant or most common alleles in certain species or populations (Torres, 1980) and also depends on the basic genome composition and the history of cultivar development. Theoretically, the number of multi-locus genotypic combinations of 11 loci identified above would be $3^{11}=$ $14,348,907$, if we assume an average of two alleles (producing three genotypes) per locus. This is far more than the currently known number of $\approx 600$ chestnut cultivars found worldwide (Liu et al., 1988; Rutter et al., 1990). As indicated in Table 6, 47 chestnut cultivars and selections can be uniquely identified by their multilocus genotypes using 12 polymorphic isozyme loci. The isozyme genetic polymorphism is quite extensive, which makes multilocus genotype identification a very powerful tool for identifying chestnut cultivars. The 47 cultivars differed on average at 7.08 loci, ranging from a difference of 1 to 12 loci. The average number of allele differences among intraspecific cultivars was $4.00 \pm 0.00$ for European chestnuts, $4.17 \pm 1.47$ for Am chestnuts, $4.17 \pm 0.98$ for Am chinquapins, $4.87 \pm 1.69$ for Ch chestnuts, $4.67 \pm 1.49$ for Japanese chestnuts, and $5.20 \pm 1.74$ for seguin chestnuts. The Japanese chestnut is closely related to the Ch chestnut with an average allelic difference of $5.58 \pm 1.32$, while between Japanese chestnut and seguin a $5.73 \pm 1.49$ allele difference was observed. Likewise, the European chestnut is closely related to the Am chestnut, as indicated by an allele difference of $6.13 \pm 0.64$ and a difference of $5.38 \pm 1.06$ alleles between European chestnut and Am chinquapin.

However, a higher degree of allozyme polymorphism is observed when comparing eastern and western species. On average, the allelic difference between $\mathrm{Ch}$ and Am cultivars is 8.70 \pm 1.19 , between $\mathrm{Ch}$ and European $9.43 \pm 1.50$, between Japanese and Am $9.36 \pm 0.99$, and between Japanese and European 9.07 \pm 1.21 . The results are in accordance with the traditional classification of chestnut species. Interestingly, the European species show more similarity to the Am species than to the Asian species. The $\mathrm{Ch}$ chestnut is considered the progenitor of both (Jaynes, 1975), giving rise to the European chestnut by westward extension and to several Am species by eastward migration. The data also suggest that the analysis of multi-locus isozyme genotypes could be very useful for plant systematics and evolution studies. Further studies are necessary to clarify the interspecific relationships and origin of the species.

\section{Literature Cited}

Anagnostakis, S.L. 1991. Peroxidase allozyme phenotypes in Castanea and their segregation among progeny. HortScience 26:1424.

Anderson, C.M., W.S. Castle, and G.A. Moore. 1991. Isozymic identification of zygotic seedlings in Swingle Citrumelo Citrus paradisi $\mathrm{x}$ Poncitrus trifoliata nursery and field populations. J. Amer. Soc. Hort. Sci. 116:322-326.

Arus, P. and T.J. Orton. 1984. Inheritance patterns and linkage relationships of eight genes of celery. J. Hered. 75:11-14.

Bernatzky, R. and S.D. Tanksley. 1986. Toward a saturated linkage map in tomato based on isozyme and random cDNA sequences. Genetics 112:887-898.

Brewbaker, J.L., C. Nagai, and E.H. Liu. 1985. Genetic polymorphisms of 13 maize peroxidase. J. Hered. 76:159-167.

Burnham, C.R. 1988. The restoration of the American Chestnut. Amer. Sci. 76:478-487.

Cerezo, M., R.S. Company, and P. Arus. 1989. Identification of almond cultivars by pollen isozymes. J. Amer. Soc. Hort. Sci. 114:164-169.

Cochran, M.F. 1990. Chestnuts-Making a comeback? Natl. Geographic 117:128-140.

Degani C., R. El-Batsri, and S. Gazit. 1990. Enzyme polymorphism in mango. J. Amer. Soc. Hort. Sci. 115:844-847.

DeWald, M.G., G.A. Moore, and W.B. Sherman. 1992. Isozymes in Ananas (pineapple): Genetics and usefulness in taxonomy. J. Amer. Soc. Hort. Sci. 117:491-196.

Garcia, P., M.P. de la Vega, and C. Benito. 1982. The inheritance of rye seed peroxidase. Theoretical Applied Genet. 61:341-351.

Gillet, E. 1991. Genetic analysis using single tree progenies. In: S. Fineschi, M.E. Malvoti, and F. Cannata (eds.). Biochemical markers in the population genetics of forest trees. SPB Acad. Publ. bv, The Hague, The Netherlands.

Gomes, J., S. Jadric, M. Winterhalter, and S. Brkic. 1982. Alcohol dehydrogenase isoenzymes in chickpea cotyledons. Phytochemistry 21:1219-1224.

Hamrick, J.L. 1989. Isozyme and analysis of genetic structure in plant populations, p. 87-105. In: D.E. Soltis and P.S. Soltis (eds.). Isozymes in plant biology. Dioscorides Press, Portland, Ore.

Hebard, F.V. 1992. Inheritance of juvenile morphological characteristics in crosses of Chinese and American Chestnut. Symp. Intl. Chestnut Conf., Morgantown, W.Va.

Hirano, H. and K. Naganuma. 1979. Inheritance of peroxidase isozymes in mulberry (Morus spp.). Euphytica 28:73-79.

Jarret, R.L. and R.E. Litz. 1986. Isozymes as genetic markers in banana and plantains. Euphytica 35:539-549.

Jaynes, R. A. 1975. Chestnuts, p. 490-499. In: J. Janick and J.N. Moore (eds.). Advances in fruit breeding. Purdue Univ. Press, West Lafayette, Ind.

Kahler, A.L. 1983. Inheritance and linkage of acid phosphatase locus Acp4 in maize. J. Hered. 74:239-246.

Liu, L., J.H. Cai, and Y.H. Zhang. 1988. Chestnut. 2nd ed. (in Chinese) Scientific Publishing House, Beijing, China.

Malvolti, M.E. and S. Fineschi. 1987. Analysis of enzyme systems in chestnut Castanea sativa Mill. Genet. Agr. 41:243-256.

Manganaris, A.G. and F.H. Alston. 1992. Genetics of esterase isozymes in Malus. Theoretical Applied Genet. 83:464-475.

Mulcahy, D.L., R.W. Robinson, M. Ihara, and R. Kesseli. 1981. Gametophytic transcription for acid phosphatase in pollen of Cucurbita species hybrids. J. Hered. 72:353-354.

Navot, N. and D. Zamir. 1986. Linkage relationships of 19 protein coding genes in watermelon. Theoretical Applied Genet. 72:274-278.

Pasteur, N., G. Pasteur, F. Bonhomme, J. Catalan, and J. Britton-Davidian. 1988. Practical isozyme genetics. Ellis Horwood Limited, New York. Quiros, C.F. and K. Morgan. 1981. Peroxidase and leucine- aminopeptidase in diploid Medicago species closely related to alfalfa: Multiple 
gene loci, multiple allelism, and linkage. Theoretical Applied Genet. 60:221-228.

Quiros, C.F. and N. McHale. 1985. Genetic analysis of isozyme variation in diploid and tetraploid potatoes. Genetics 111:131-135.

Rick, C.M., R.W.Zobel, and J.F. Fobes. 1974. Four peroxidase loci in redfruited tomato species: genetics and geographic distribution. Proc. Natl. Acad. Sci. USA 71:835-839.

Rutter, P.A., G. Miller, and J.A. Payne. 1990. Chestnuts, p. 761-788. In: J.N. Moore and J.R. Ballington Jr. (eds.). Genetic resources of temperate fruit and nut crops. Intl. Soc. Hort. Sci., Wageningen, the Netherlands.

Sawano, M., T. Ichii, T. Nakanish, and Z. Kotera. 1984. Study on identification of chestnut species and varieties by isozyme analysis. Sci. Rpt. Fac. Agr. Kobe Univ. 16:67-71.

Suiter, K.A. 1988. Genetics of allozyme variation in Gossypium arboreum L. and Gossypium herbaceum L. (Malvaceae). Theoretical Applied Genet. 75:259-271.

Suiter, K.A., J.F. Wendel, and J.S. Case. 1983. LINKAGE-1: A PASCAL computer program for the detection and analysis of genetic linkage. J. Hered. 74:203-204.

Tanksley, S.D. 1984. Linkage relationships and chromosomal locations of enzyme-coding genes in pepper Capsicum annuum. Chromosoma 89:352-360.

Tanksley, S.D. and T.J. Orton (eds.). 1983. Isozymes in plant genetics and breeding, part B. Elsevier, Amsterdam.

Tanksley, S.D. and C.M. Rick. 1980a. Genetics of esterase in species of Lycopersicon. Theoretical Applied Genet. 56:209-219.

Tanksley, S.D. and C.M. Rick. 1980b. Isozyme gene linkage map of the tomato: Applications in genetics and breeding. Theoretical Applied Genet. 57:161-170.

Torres, A.M. 1974. Genetics of sunflower alcohol dehydrogenase: $A d h_{2}$ nonlinkage to $A d h h_{1}$ and $A d h$ early alleles. Biochem. Genet. 12:385-395.

Torres, A.M. 1980. Fruit and leaf isozyme as genetic markers in avocado. J. Amer. Soc. Hort. Sci. 105:614-619.
Torres, A.M., T. Mau-Lastovicka, T.E. Williams, and R.K. Soost. 1985. Segregation distortion and linkage of Citrus and Poncirus isozyme genes. J. Hered. 76:289-294.

Torres, A.M., R.K. Soost, and T. Mau-Lastovicka. 1982. Citrus isozymes: Genetics and distinguishing nucellar from zygotic seedlings. J. Hered. 73:335-339.

van den Berg, B.M and H.J.W. Wijsman. 1982. Genetics of peroxidase isozyme in Petunia. Theoretical Applied Genet. 63:33-38.

Weeden, N.F. and L.D. Gottlieb. 1980. The genetics of chloroplast enzymes. J. Hered. 71:392-396.

Weeden, N.F. and R.C. Lamb. 1985. Identification of apple cultivars by isozyme phenotypes. J. Amer. Soc. Hort. Sci. 110:509-515.

Weeden, N.F. and R.C. Lamb. 1987. Genetics and linkage analysis of 19 isozyme loci in apple. J. Amer. Soc. Hort. Sci. 112:865-872.

Weeden, N.F. and G.A. Marx. 1987. Further genetic analysis and linkage relationships of isozyme loci in the pea. J. Hered. 78:153-159.

Weeden, N.F., B.I. Reisch, and M.E. Martens. 1988. Genetic analysis of isozyme polymorphism in grape. J. Amer. Soc. Hort. Sci. 113:765-769.

Weeden, N.F. and R.W. Robinson. 1986. Allozyme segregation ratio in the interspecific cross Cucurbita maxiam $\times$ C. ecuadorensis suggest that hybrid breakdown is not caused by minor alterations in chromosome structure. Genetics 114:593-609.

Wendel, J.F. and C.R. Parks. 1982. Genetic control of isozyme variation in Camellia japonica L. J. Hered. 73:197-204.

Wendel, J.F., M.M. Goodman, C.W. Stuber, and J.B. Beckett. 1988. New isozyme systems for maize (Zea mays L.): Aconitate hydratase, adenylate kinase, NADH dehydrogenase, and shikimate dehydrogenase. Biochem. Genet. 26:421-445.

Wendel, J.F. and N.F. Weeden. 1989. Visualization and interpretation of plant isozymes, p. 9-33. In: D.E. Soltis and P.S. Soltis (eds.). Isozymes in plant biology. Dioscorides Press, Portland, Ore.

Yates, F. 1934. Contingency tables involving small numbers and the $\chi^{2}$. J. Royal Stat. Soc. Suppl. 1:217-235. 